\title{
WEAK WEYL'S LAW FOR CONGRUENCE SUBGROUPS*
}

\author{
JEAN-PIERRE LABESSE† AND WERNER MÜLLER ${ }^{\ddagger}$
}

1. Introduction. Let $G$ be a connected and simply connected semisimple algebraic group defined over $\mathbb{Q}$ and let $Z_{G}$ be its center. Let $\Gamma$ be an arithmetic subgroup of $G(\mathbb{R})$. For simplicity we assume in the introduction that $\Gamma$ is torsion free. We denote by $K_{\infty}$ a maximal compact subgroup of $G(\mathbb{R})$. We endow $G(\mathbb{R})$ and $K_{\infty}$ with Haar measures so that the symmetric space $X=G(\mathbb{R}) / K_{\infty}$ has the canonical measure, i.e., the measure defined using the Killing form. Let

$$
H_{\text {cusp }}^{\Gamma}=L_{\text {cusp }}^{2}(\Gamma \backslash G(\mathbb{R}))
$$

denote the closure in $L^{2}(\Gamma \backslash G(\mathbb{R}))$ of the subspace spanned by all $\Gamma$-cuspidal automorphic forms. We denote by $\rho_{\infty}$ the right regular representation of $G(\mathbb{R})$ in $H_{\text {cusp }}^{\Gamma}$. Let $\left(\sigma, V_{\sigma}\right)$ be an irreducible unitary representation of $K_{\infty}$. Set

$$
H_{\text {cusp }}^{\Gamma}(\sigma)=\left(H_{\text {cusp }}^{\Gamma} \otimes V_{\sigma}\right)^{K_{\infty}} .
$$

Let $\Omega$ be the Casimir operator for $G(\mathbb{R})$. Then $\rho_{\infty}(\Omega) \otimes$ Id induces a selfadjoint operator in the Hilbert space $H_{\text {cusp }}^{\Gamma}(\sigma)$ which has pure point spectrum. We denote by $\Lambda_{\text {cusp }}(\sigma)$ the set of eigenvalues of $\rho_{\infty}(\Omega) \otimes \operatorname{Id}$ in $H_{\text {cusp }}^{\Gamma}(\sigma)$. Let $N_{\text {cusp }}^{\Gamma}(T, \sigma)$ be the counting function of the cuspidal spectrum $\Lambda_{\text {cusp }}(\sigma)$, i.e., $N_{\text {cusp }}^{\Gamma}(T, \sigma)$ is the number of eigenvalues $\lambda$ of $\rho_{\infty}(\Omega) \otimes \operatorname{Id}$ in $H_{\text {cusp }}^{\Gamma}(\sigma)$, counted with their multiplicities, with $|\lambda| \leq T$. We shall also consider the discrete subspace

$$
H_{\text {disc }}^{\Gamma}=L_{\text {disc }}^{2}(\Gamma \backslash G(\mathbb{R})),
$$

and we define $H_{\text {disc }}^{\Gamma}(\sigma)$ and the corresponding eigenvalue counting function $N_{\text {disc }}^{\Gamma}(T, \sigma)$ similarly. It is an important question in the theory of automorphic forms to understand the asymptotic behavior of the counting function as $T \rightarrow \infty$. Let $d$ be the dimension of the symmetric space $X=G(\mathbb{R}) / K_{\infty}$ and let $\Gamma$ denote the Gamma function. Let

$$
c_{\sigma}(\Gamma)=\frac{\operatorname{dim}(\sigma) \operatorname{vol}(\Gamma \backslash X)}{(4 \pi)^{d / 2} \boldsymbol{\Gamma}(d / 2+1)}
$$

be Weyl's constant. A conjecture of Sarnak [Sa] states that the counting function of the cuspidal spectrum satisfies Weyl's law, i.e.,

$$
\lim _{T \rightarrow \infty} \frac{N_{\text {cusp }}^{\Gamma}(T, \sigma)}{T^{d / 2}}=\lim _{T \rightarrow \infty} \frac{N_{\text {disc }}^{\Gamma}(T, \sigma)}{T^{d / 2}}=c_{\sigma}(\Gamma) .
$$

This conjecture has been established in some special cases. First of all, it was Selberg [Se] who proved it for congruence subgroups of $\operatorname{SL}(2, \mathbb{Z})$ and $\sigma=1$. Other cases for which the conjecture has been established are Hilbert modular groups [Ef],

\footnotetext{
*Received February 16, 2004; accepted for publication April 15, 2004.

† Institut de Mathématiques de Luminy, Université Aix-Marseille II, Av. de Luminy, Case Postale 907, 13288 Marseille, CEDEX 9, France (labesse@iml.univ-mrs.fr).

${ }^{\ddagger}$ Universität Bonn, Mathematisches Institut, Beringstrasse 1, D-53115 Bonn, Germany (mueller@math.uni-bonn.de).
} 
congruence subgroups of $\mathrm{SO}(n, 1)[\mathrm{Rez}], \mathrm{SL}(3, \mathbb{Z})$ [Mil], and in particular, the conjecture was proved in $[\mathrm{Mu} 2]$ for principal congruence subgroups of $\mathrm{SL}(n, \mathbb{Z})$ and arbitrary $\sigma$.

A possible approach to prove (1.1) is through the Selberg trace formula applied to a function constructed from the heat kernel of the corresponding Bochner-Laplace operator. Let $E_{\sigma} \rightarrow X$ be the homogeneous vector bundle associated to $\sigma$ and let $\Delta_{\sigma}$ be the elliptic differential operator induced by $-R(\Omega) \otimes \operatorname{Id}$ in $C^{\infty}\left(X, E_{\sigma}\right)$, where $R$ denotes the right regular representation of $G(\mathbb{R})$ in $C^{\infty}(G(\mathbb{R}))$. Using the heat kernel of $\Delta_{\sigma}$, one produces a 1-parameter family of functions $h_{t}$ on $G(\mathbb{R})$, depending on $t>0$, such that for any irreducible unitary representation $(\pi, \mathcal{H}(\pi))$ of $G(\mathbb{R})$ one has

$$
\operatorname{Tr} \pi\left(h_{t}\right)=e^{t \pi(\Omega)} \operatorname{dim} \operatorname{Hom}_{K_{\infty}}\left(\mathcal{H}(\pi), V_{\sigma}^{*}\right) .
$$

Given $\lambda \in \Lambda_{\text {cusp }}(\sigma)$, let $m(\lambda)$ denote its multiplicity. Then it follows from (1.2) that

$$
\operatorname{Tr}\left(\rho_{\infty}\left(h_{t}\right) \mid H_{\text {cusp }}^{\Gamma}\right)=\sum_{\lambda \in \Lambda_{\text {cusp }}(\sigma)} m(\lambda) e^{t \lambda} .
$$

The idea is now to study the asymptotic behavior of the left hand side as $t \rightarrow 0$. Using the properties of the heat kernel, it follows that

$$
h_{t}(1) \sim \frac{\operatorname{dim}(\sigma)}{(4 \pi t)^{d / 2} \operatorname{vol}\left(K_{\infty}\right)}
$$

as $t \rightarrow 0$. A good control of the trace formula should allow to prove that as $t \rightarrow 0$, the constant term in the asymptotic expansion of

$$
t \longmapsto t^{d / 2} \operatorname{Tr}\left(\rho\left(h_{t}\right) \mid H_{\text {cusp }}^{\Gamma}\right)
$$

is the contribution of the unit element to the trace formula:

$$
t^{d / 2} \operatorname{Tr}\left(\rho\left(h_{t}\right) \mid H_{\text {cusp }}^{\Gamma}\right)=t^{d / 2} \operatorname{vol}(\Gamma \backslash G(\mathbb{R})) h_{t}(1)+o(1)
$$

(we recall that we have assumed that $\Gamma$ is torsion free). The desired estimate is then an immediate consequence of (1.3) combined with the Tauberian theorem. The second named author has been able to apply successfully this method for $\Gamma$ a principal congruence subgroup for $G=\mathrm{SL}(n)$. However, many technical difficulties are still to overcome in order to prove (1.1) for a general $G$.

The purpose of this paper is to prove a weaker result which holds for every $G$. Recall that an upper bound, which holds for arbitrary $G$ and $\Gamma$, is already known thanks to Donnelly [Do]:

$$
\limsup _{T \rightarrow \infty} \frac{N_{\text {cusp }}^{\Gamma}(T, \sigma)}{T^{d / 2}} \leq c_{\sigma}(\Gamma)
$$

By working with a simple form of the trace formula, we shall get, for a general $G$, a lower bound that depends on the choice of a set $S$ of primes containing at least two finite primes. For every such set $S$ we shall define a certain constant $c_{S}(\Gamma) \leq 1$, which is non zero for $\Gamma$ deep enough. Let $Z_{\Gamma}=\Gamma \cap Z_{G}(\mathbb{R})$. Note that $Z_{\Gamma} \subset K_{\infty}$. Then our main result is the following theorem. 
THEOREM 1.1. Let $G$ be an almost simple connected and simply connected semisimple algebraic group defined over $\mathbb{Q}$ such that $G(\mathbb{R})$ is non compact. Then for every congruence subgroup $\Gamma$ and every $\sigma$ such that $\left.\sigma\right|_{Z_{\Gamma}}=\operatorname{Id}$ we have

$$
c_{\sigma}(\Gamma) c_{S}(\Gamma) \leq \liminf _{T \rightarrow \infty} \frac{N_{\mathrm{cusp}}^{\Gamma}(T, \sigma)}{T^{d / 2}} .
$$

This will turn out to be very easy to prove but the price to pay for simplicity is that this lower bound is far from being sharp; in particular the lower bound is non trivial, i.e. $c_{S}(\Gamma)>0$, only for discrete subgroups $\Gamma$ that are deep enough (see below for a more precise statement). We note that a weaker form of this result is due to Piatetski-Shapiro [PS] and for $G=\mathrm{SL}(2, \mathbb{R})$ also to Venkov [Ve]. In [PS] it is proved that for every $\Gamma$ there exists a subgroup of finite index $\Gamma^{\prime}$ such that the space of Maass cusp forms with respect to $\Gamma^{\prime}$ is infinite dimensional.

Theorem 1.1 is a consequence of Theorem 4.2. To get the lower bound we shall prove a sharp estimate for a certain strict subspace $H_{\text {cusp }}^{\Gamma, S}$ of $H_{\text {cusp }}^{\Gamma}$ that will be defined via the adèlic picture. From now on, for simplicity of exposition, let us assume that $G$ is absolutely almost simple and that $G(\mathbb{R})$ is non compact. We consider a discrete subgroup $\Gamma$ such that

$$
\Gamma=K_{\text {fin }} \cap G(\mathbb{Q}),
$$

where

$$
K_{\text {fin }}=\prod K_{p}
$$

is a decomposable open compact subgroup of the group of finite adèles $G\left(\mathbb{A}_{\mathrm{fin}}\right)$. Let $S$ be a finite set of primes of cardinality at least 2 and let

$$
L_{\text {cusp }}^{2}(G(\mathbb{Q}) \backslash G(\mathbb{A}), S) \subset L_{\text {cusp }}^{2}(G(\mathbb{Q}) \backslash G(\mathbb{A}))
$$

be the $G(\mathbb{A})$-module generated by cusp forms that are orthogonal to the trivial representation of $G(\mathbb{A})$ and on which $G_{S}=G\left(\mathbb{Q}_{S}\right)$ acts by the Steinberg representation. Then we define

$$
H_{\text {cusp }}^{\Gamma, S}=L_{\text {cusp }}^{2}(G(\mathbb{Q}) \backslash G(\mathbb{A}), S)^{K_{\text {fin }}}
$$

and

$$
H_{\text {cusp }}^{\Gamma}(\sigma, S)=\left(L_{\text {cusp }}^{2}(G(\mathbb{Q}) \backslash G(\mathbb{A}), S) \otimes V_{\sigma}\right)^{K}=\left(H_{\text {cusp }}^{\Gamma, S} \otimes V_{\sigma}\right)^{K_{\infty}} .
$$

Let $\Lambda_{\text {cusp }}(\sigma, S)$ be the spectrum of $\rho_{\infty}(\Omega) \otimes \operatorname{Id}$ in $H_{\text {cusp }}^{\Gamma}(\sigma, S)$. It consists of a discrete set of eigenvalues. Given $\lambda \in \Lambda_{\text {cusp }}(\sigma, S)$ denote by $m(\lambda)$ the multiplicity of the eigenvalue $\lambda$. Then it follows from (1.2) that

$$
\operatorname{Tr}\left(\rho_{\infty}\left(h_{t}\right) \mid H_{\text {cusp }}^{\Gamma, S}\right)=\sum_{\lambda \in \Lambda_{\operatorname{cusp}}(\sigma, S)} m(\lambda) e^{t \lambda} .
$$

Using the trace formula, we shall prove in 4.2 that

$$
\operatorname{Tr}\left(\rho_{\infty}\left(h_{t}\right) \mid H_{\text {cusp }}^{\Gamma, S}\right) \sim c_{S}(\Gamma) \frac{\operatorname{dim} \sigma \operatorname{vol}(\Gamma \backslash X)}{(4 \pi t)^{d / 2}}
$$


as $t \rightarrow 0$. For $T \geq 0$ let $\Lambda_{\text {cusp }}(\sigma, S ; T)$ be the subset of all $\lambda \in \Lambda_{\text {cusp }}(\sigma, S)$ with $|\lambda| \leq T$. Set

$$
N_{\text {cusp }}^{\Gamma}(T, \sigma, S)=\sum_{\lambda \in \Lambda_{\operatorname{cusp}}(\sigma, S ; T)} m(\lambda)
$$

If $c_{S}(\Gamma) \neq 0$ the Tauberian theorem yields the estimate:

$$
\lim _{T \rightarrow \infty} \frac{N_{\text {cusp }}^{\Gamma}(T, \sigma, S)}{T^{d / 2}}=c_{\sigma}(\Gamma) c_{S}(\Gamma) .
$$

This is our Theorem 4.2. Now clearly $N_{\text {cusp }}^{\Gamma}(T, \sigma, S) \leq N_{\text {cusp }}^{\Gamma}(T, \sigma)$ and hence

$$
c_{\sigma}(\Gamma) c_{S}(\Gamma) \leq \liminf _{T \rightarrow \infty} \frac{N_{\mathrm{cusp}}^{\Gamma}(T, \sigma)}{T^{d / 2}} .
$$

We still have to observe that $c_{S}(\Gamma) \neq 0$ for subgroups $\Gamma$ such that at primes $p \in S$ the open compact subgroup $K_{p}$ is small enough, i.e., if it is a subgroup of some minimal parahoric subgroup $I_{p}$ of $G_{p}=G\left(\mathbb{Q}_{p}\right)$.

2. A simple trace formula. From now on we shall work in the adèlic picture. We choose a Haar measure, for example the Tamagawa measure, on $G(\mathbb{A})$ which is given as the product of measures on each $G_{p}=G\left(\mathbb{Q}_{p}\right)$. We fix a decomposable open compact subgroup $K_{\text {fin }}$ of $G\left(\mathbb{A}_{\text {fin }}\right)$. Set

$$
K=K_{\infty} K_{\text {fin }} .
$$

Then $K$ is a compact subgroup of $G(\mathbb{A})$. Let $f \in C_{c}^{\infty}(G(\mathbb{A}))$ be $K$-finite. The Arthur-Selberg trace formula in its invariant form is the equality

$$
\sum_{\mathfrak{o} \in \mathcal{O}} I_{\mathfrak{o}}(f)=I(f)=\sum_{\chi \in \mathfrak{X}} I_{\chi}(f)
$$

of a geometric and a spectral expansion for an invariant linear form $f \longmapsto I(f)$ (see $[\mathrm{Ar}])$.

Let $\Sigma$ be some large enough set of primes, containing in particular the real place and all ramified primes. We shall consider functions $f$ that are decomposable

$$
f=\otimes f_{p}
$$

and such that for $p \notin \Sigma, f_{p}$ is the characteristic function of a hyperspecial maximal compact subgroup divided by its volume. We put, as usual,

$$
f^{\Sigma}=\underset{p \notin \Sigma}{\otimes} f_{p}
$$

and

$$
f_{\Sigma}=\underset{p \in \Sigma}{\otimes} f_{p}
$$

so that $f=f_{\Sigma} \otimes f^{\Sigma}$. The main observation is the following result of Arthur: when applied to a decomposable function such that $f_{p}$ is cuspidal, in the sense of Arthur, at two primes $p \in S$ the trace formula is "simple". 
Proposition 2.1. If $f$ is cuspidal at two places, the linear form $I(f)$ has a discrete spectral expansion:

$$
I(f)=\sum a(\pi) \operatorname{tr} \pi(f)
$$

where the sum is over certain subrepresentations of parabolically induced representations from representations in the discrete spectrum of Levi subgroups. In particular, if $\pi$ occurs in the discrete spectrum for $G(\mathbb{A})$ then $a(\pi)=m(\pi)$, where $m(\pi)$ denotes the multiplicity with which $\pi$ occurs in the discrete spectrum. The geometric expansion is given by a linear combination of ordinary orbital integrals (over $G_{\Sigma}$ )

$$
I(f)=\sum_{\gamma \in \mathfrak{G}_{\Sigma}} a(\gamma, \Sigma) \mathcal{O}_{\gamma}\left(f_{\Sigma}\right)
$$

where $\mathfrak{G}_{\Sigma}$ is a set of representatives of conjugacy classes with elliptic semisimple part, $\mathcal{O}_{\gamma}\left(f_{\Sigma}\right)$ is the orbital integral of $f_{\Sigma}$ and $a(\gamma, \Sigma)$ is some scalar. If $\gamma$ is elliptic we have

$$
a(\gamma, \Sigma) \mathcal{O}_{\gamma}\left(f_{\Sigma}\right)=a(\gamma) \mathcal{O}_{\gamma}(f),
$$

where the scalar a $(\gamma)$ is independent of $\Sigma$. Moreover if the support of $f_{\Sigma}$ remains in a fixed compact set then, in the sum over $\mathfrak{G}_{\Sigma}$, only a finite number of elliptic conjugacy classes in $G(\mathbb{Q})$ may give a non trivial contribution.

Proof. This is the content of [Ar, Corollary 7.2]. We omit the sum over $t \geq 0$ that occurs in [Ar] since the sum is now known to be absolutely convergent by [Mu1].

We shall denote by $1(f)$ the operator defined by $f$ in the trivial representation; this is a scalar operator in a one dimensional space:

$$
1(f)=\int_{G(\mathbb{A})} f(x) d x
$$

Let $p$ be a finite place. The existence of (normalized) pseudo-coefficients of the Steinberg representation is a particular case of a general result due to Kazdan, but, up to a sign, they can also be viewed as Euler-Poincaré functions at non archimedean places and an explicit construction has been given by Kottwitz [Ko]. A pseudo-coefficient of the Steinberg representation is a $K_{p}$-finite function $f_{p} \in C_{c}^{\infty}\left(G_{p}\right)$ such that

$$
\operatorname{Tr} \pi_{p}\left(f_{p}\right)=0
$$

for all irreducible unitary representations $\pi_{p}$ unless either $\pi_{p}$ is the Steinberg representation of $G_{p}$ in which case

$$
\operatorname{Tr} \pi_{p}\left(f_{p}\right)=1
$$

or $\pi_{p}=1_{p}$ the trivial representation in which case

$$
1_{p}\left(f_{p}\right)=(-1)^{q}
$$

for some integer $q$. Moreover, [Ko, Theorem 2] shows that orbital integrals $\mathcal{O}_{\gamma}\left(f_{p}\right)$ of non elliptic elements $\gamma$ vanish.

Let $S$ be a finite set of finite primes. Let

$$
L_{\text {cusp }}^{2}(G(\mathbb{Q}) \backslash G(\mathbb{A}), S) \subset L_{\text {cusp }}^{2}(G(\mathbb{Q}) \backslash G(\mathbb{A}))
$$


be the $G(\mathbb{A})$-module generated by cusp forms that are orthogonal to the trivial representation of $G(\mathbb{A})$ and on which

$$
G_{S}:=\prod_{p \in S} G\left(\mathbb{Q}_{p}\right)
$$

acts by the Steinberg representation. Let $\rho$ denote the regular representation of $G(\mathbb{A})$ in $L_{\text {cusp }}^{2}(G(\mathbb{Q}) \backslash G(\mathbb{A}))$.

Proposition 2.2. Let $G$ be a simply connected almost simple algebraic group defined over $\mathbb{Q}$. Assume that $S$ is a set of finite primes containing at least two primes. Consider

$$
f=f_{\infty} \otimes f_{\text {fin }, S} \otimes f_{S},
$$

where $f_{\infty} \in C_{c}^{\infty}\left(G_{\infty}\right)$, $f_{\text {fin }, S}$ is a locally constant compactly supported function on $G\left(\mathbb{A}_{\text {fin }}^{S}\right)$, the group of finite adèles outside $S$, and $f_{S}$ is a pseudo-coefficient of the Steinberg representation of $G_{S}$. Then one has

$$
\sum_{\gamma \in \mathfrak{G}_{e}} a(\gamma) \mathcal{O}_{\gamma}(f)=\operatorname{Tr}\left(\rho(f) \mid L_{\text {cusp }}^{2}(G(\mathbb{Q}) \backslash G(\mathbb{A}), S)\right)+1(f),
$$

where $\mathfrak{G}_{e}$ is a set of representatives of elliptic conjugacy classes in $G(\mathbb{Q})$.

Proof. By a standard argument, automorphic representations in the discrete spectrum with a Steinberg component at some place $p$ must be cuspidal. In fact Langlands description of the discrete spectrum implies that representations that are in the non cuspidal discrete spectrum arise from residues of Eisenstein series for non unitary parameters and hence are non tempered at all places, while the Steinberg representation is tempered [Clo, Proposition 4.10]. Moreover, automorphic representations with a Steinberg component at some place $p$ cannot be subrepresentations of parabolically induced representations from representations in the discrete spectrum of Levi subgroups for a proper parabolic subgroup. Suppose that $G_{p}$ is non compact. Since our group $G$ is absolutely almost simple and simply connected, the strong approximation theorem holds and hence $G(\mathbb{Q}) G_{p}$ is dense in $G(\mathbb{A})$. Therefore an automorphic representations that is trivial at $p$ is necessarily trivial. On the other hand, if the group $G_{p}$ is compact then all automorphic representations are cuspidal. Hence an automorphic representations that is trivial at one place is either trivial or cuspidal. We now use Proposition 2.1 on the simple form of the trace formula with $\Sigma$ large enough, containing $S$. The above discussion shows that the spectral expansion of $I(f)$ is given by the sum of the trace in the cuspidal spectrum plus the trace in the trivial representation:

$$
I(f)=\operatorname{Tr}\left(\rho(f) \mid L_{\text {cusp }}^{2}(G(\mathbb{Q}) \backslash G(\mathbb{A}), S)\right)+1(f) .
$$

The geometric expansion of $I(f)$ is a linear combination of orbital integrals (over $G_{\Sigma}$ ) and, as recalled above, [Ko, Theorem 2] shows that orbital integrals of non elliptic elements vanish at $p \in S$. Hence

$$
I(f)=\sum_{\gamma \in \mathfrak{G}_{e}} a(\gamma) \mathcal{O}_{\gamma}(f),
$$

where $\mathfrak{G}_{e}$ is a set of representatives of elliptic conjugacy classes in $G(\mathbb{Q})$. 
Let

$$
H_{\text {cusp }}^{\Gamma, S}=L_{\text {cusp }}^{2}(G(\mathbb{Q}) \backslash G(\mathbb{A}), S)^{K_{\text {fin }}}
$$

be the Hilbert subspace of $L_{\text {cusp }}^{2}(G(\mathbb{Q}) \backslash G(\mathbb{A}))$ generated by vectors of automorphic

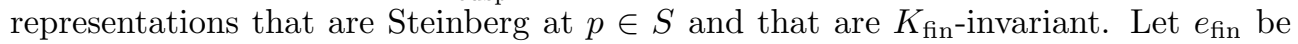
the characteristic function of $K_{\text {fin }}$, our chosen open compact subgroup at finite adèles divided by its volume:

$$
e_{\text {fin }}=\frac{1}{\operatorname{vol}\left(K_{\text {fin }}\right)} \chi_{K_{\text {fin }}}
$$

Let

$$
K_{S}=\prod_{p \in S} K_{p}, \quad K_{\mathrm{fin}, S}=\prod_{p \notin S \cup\{\infty\}} K_{p} .
$$

Let $e_{S}\left(\right.$ resp. $\left.e_{\mathrm{fin}, S}\right)$ be the characteristic function of $K_{S}$ (resp. $\left.K_{\mathrm{fin}, S}\right)$ divided by its volume. Then

$$
e_{\mathrm{fin}}=e_{S} \otimes e_{\mathrm{fin}, S}
$$

Corollary 2.3. Assume that $f$ is of the form

$$
f=f_{\infty} \otimes f_{S} \otimes e_{\text {fin }, S}
$$

where $f_{S}$ is a pseudo-coefficient of the Steinberg representation of $G_{S}$. Let $c\left(K_{S}\right)$ be the dimension of the space of $K_{S}$ fixed vectors in the Steinberg representation of $G_{S}$ and let

$$
b\left(f_{\infty}, S\right)=\operatorname{Tr} 1\left(f_{\infty} \otimes f_{S}\right),
$$

where 1 is the trivial representation of $G_{\infty} \times G_{S}$. Then one has

$$
\operatorname{Tr}\left(\rho_{\infty}\left(f_{\infty}\right) \mid H_{\text {cusp }}^{\Gamma, S}\right)=c\left(K_{S}\right)\left(\sum_{\gamma \in \mathfrak{G}_{e}} a(\gamma) \mathcal{O}_{\gamma}(f)-b\left(f_{\infty}, S\right)\right) .
$$

Proof. We first observe that for any admissible representation $\tau$ of $G\left(\mathbb{A}_{\mathrm{fin}}\right)$, the operator $\tau\left(e_{\text {fin }}\right)$ is the projection on the subspace of $K_{\text {fin }}$-invariant vectors. Hence,

$$
\operatorname{Tr}\left(\rho_{\infty}\left(f_{\infty}\right) \mid H_{\text {cusp }}^{\Gamma, S}\right)=\operatorname{Tr}\left(\rho\left(f_{\infty} \otimes e_{\text {fin }}\right) \mid L_{\text {cusp }}^{2}(G(\mathbb{Q}) \backslash G(\mathbb{A}), S)\right) .
$$

However, if $\pi_{S}$ is the Steinberg representation of $G_{S}$ then

$$
\operatorname{Tr} \pi_{S}\left(e_{S}\right)=c\left(K_{S}\right) \operatorname{Tr} \pi_{S}\left(f_{S}\right)
$$

and hence we get

$$
\operatorname{Tr}\left(\rho_{\infty}\left(f_{\infty}\right) \mid H_{\text {cusp }}^{\Gamma, S}\right)=c\left(K_{S}\right) \operatorname{Tr}\left(\rho(f) \mid L_{\text {cusp }}^{2}(G(\mathbb{Q}) \backslash G(\mathbb{A}), S)\right) .
$$

The assertion is then an immediate consequence of Proposition 2.2. 
3. The heat kernel. In this section we establish a number of facts about the heat kernel of a Bochner-Laplace operator on the Riemannian symmetric space $X=$ $G(\mathbb{R}) / K_{\infty}$. Let $E_{\sigma} \rightarrow X$ be the homogeneous vector bundle associated to $\sigma$. Let $\Omega \in$ $\mathcal{Z}\left(\mathfrak{g}_{\mathbb{C}}\right)$ be the Casimir element of $G(\mathbb{R})$ and let $R$ be the right regular representation of $G(\mathbb{R})$ on $C^{\infty}(G(\mathbb{R}))$. Denote by $\Delta_{\sigma}$ the second order elliptic differential operator induced by $-R(\Omega) \otimes \operatorname{Id}$ in $C^{\infty}\left(X, E_{\sigma}\right) \cong\left(C^{\infty}(G(\mathbb{R})) \otimes V_{\sigma}\right)^{K_{\infty}}$. If $\nabla^{\sigma}$ denotes the canonical invariant connection on $E_{\sigma}$, then

$$
\Delta_{\sigma}=\left(\nabla^{\sigma}\right)^{*} \nabla^{\sigma}-\lambda_{\sigma} \mathrm{Id},
$$

where $\lambda_{\sigma}=\sigma\left(\Omega_{K_{\infty}}\right)$ is the Casimir eigenvalue of $\sigma$. Hence $\Delta_{\sigma}: C_{c}^{\infty}\left(X, E_{\sigma}\right) \rightarrow$ $L^{2}\left(X, E_{\sigma}\right)$ is essentially selfadjoint and bounded from below. We continue to denote its unique selfadjoint extension by $\Delta_{\sigma}$. Let $\exp \left(-t \Delta_{\sigma}\right), t \geq 0$, be the associated heat semigroup. The heat operator is a smoothing operator on $L^{2}\left(X, E_{\sigma}\right)$ which commutes with the representation of $G(\mathbb{R})$ on $L^{2}\left(X, E_{\sigma}\right)$. Therefore, it is of the form

$$
\left(e^{-t \Delta_{\sigma}} \varphi\right)(g)=\int_{G(\mathbb{R})} H_{t}\left(g^{-1} g_{1}\right)\left(\varphi\left(g_{1}\right)\right) d g_{1}, \quad g \in G(\mathbb{R}),
$$

where $\varphi \in\left(L^{2}(G(\mathbb{R})) \otimes V_{\sigma}\right)^{K_{\infty}}$ and $H_{t}: G(\mathbb{R}) \rightarrow \operatorname{End}\left(V_{\sigma}\right)$ is in $L^{2} \cap C^{\infty}$ and satisfies the covariance property

$$
H_{t}(g)=\sigma(k) H_{t}\left(k^{-1} g k^{\prime}\right) \sigma\left(k^{\prime}\right)^{-1}, \quad \text { for } g \in G(\mathbb{R}), k, k^{\prime} \in K_{\infty} .
$$

Let $\mathcal{C}^{1}(G(\mathbb{R}))$ be Harish-Chandra's space of integrable rapidly decreasing functions on $G(\mathbb{R})$. Then

$$
H_{t} \in\left(\mathcal{C}^{1}(G(\mathbb{R})) \otimes \operatorname{End}\left(V_{\sigma}\right)\right)^{K_{\infty} \times K_{\infty}}
$$

[BM, Proposition 2.4].

Next we need to estimate the covariant derivatives of the heat kernel. Let $\nabla$ denote the Levi-Civita connection on $G(\mathbb{R})$ with respect to the invariant metric on $G(\mathbb{R})$. Let $d(x, y)$ be the geodesic distance of $x, y \in X$. Define the function $r(g)$ on $G(\mathbb{R})$ by

$$
r(g)=d\left(g K_{\infty}, K_{\infty}\right), \quad g \in G(\mathbb{R}) .
$$

The following proposition is proved in [Mu2, Proposition 2.1].

Proposition 3.1. Let $a=\operatorname{dim} G(\mathbb{R}), l \in \mathbb{N}_{0}$ and $T>0$. There exist $C, c>0$ such that

$$
\left\|\nabla^{l} H_{t}(g)\right\| \leq C t^{-(a+l) / 2} \exp \left(-\frac{c r^{2}(g)}{t}\right)
$$

for all $0<t \leq T$ and $g \in G(\mathbb{R})$.

Define the one-parameter family of smooth functions $h_{t}$ on $G(\mathbb{R})$ by

$$
h_{t}(g)=\operatorname{tr} H_{t}(g), \quad g \in G(\mathbb{R}), t \geq 0,
$$

where $\operatorname{tr}$ denotes the trace function on $\operatorname{End}\left(V_{\sigma}\right)$. Then it follows from 3.1 that $h_{t}$ belongs to $\mathcal{C}^{1}(G(\mathbb{R}))$. In addition, $h_{t}$ is both left and right $K_{\infty}$-finite.

Let $\pi$ be a unitary irreducible representation of $G(\mathbb{R})$ on the Hilbert space $\mathcal{H}(\pi)$. Then $\pi\left(h_{t}\right)$ is trace class and it follows as in $[\mathrm{BM}]$ that

$$
\operatorname{Tr} \pi\left(h_{t}\right)=e^{t \pi(\Omega)} \operatorname{dim} \operatorname{Hom}_{K_{\infty}}\left(\mathcal{H}(\pi), V_{\sigma}^{*}\right) .
$$


4. The main result. First we note that by our assumptions on G, strong approximation holds for $G(\mathbb{A})$ and hence we have

$$
L_{\text {cusp }}^{2}\left(G(\mathbb{Q}) \backslash G(\mathbb{A}) / K_{\text {fin }}\right) \cong L_{\text {cusp }}^{2}(\Gamma \backslash G(\mathbb{R}))
$$

as $G(\mathbb{R})$-modules. Let $h_{t}, t>0$, be the one-parameter family of functions on $G(\mathbb{R})$ defined by (3.3). By [Mu1, Corollary 0.2] the restriction of $\rho_{\infty}\left(h_{t}\right)$ to $L_{\text {cusp }}^{2}(\Gamma \backslash G(\mathbb{R}))$ is a trace class operator. However, since the support of $h_{t}$ is not compact it is not known that we may insert $h_{t}$ in the trace formula for an arbitrary $G$ (although this is likely since $h_{t}$ is a very rapidly decreasing function at infinity). This difficulty is bypassed by modifying $h_{t}$ in the following way. Let $\varphi \in C_{c}^{\infty}(\mathbb{R})$ be such that $\varphi(u)=1$, if $|u| \leq 1 / 2$, and $\varphi(u)=0$, if $|u| \geq 1$. Given $t>0$, let $\varphi_{t} \in C_{c}^{\infty}(G(\mathbb{R}))$ be defined by

$$
\varphi_{t}(g)=\varphi\left(r^{2}(g) / t^{1 / 2}\right)
$$

where $r(g)$ is defined by (3.2). Set

$$
\tilde{h}_{t}(g)=\varphi_{t}(g) h_{t}(g), \quad g \in G(\mathbb{R}), t>0 .
$$

Then $\tilde{h}_{t} \in C_{c}^{\infty}(G(\mathbb{R}))$. Recall that $\rho_{\infty}$ denotes the right regular representation of $G(\mathbb{R})$ in $H_{\text {cusp }}^{\Gamma}$.

Proposition 4.1. There exist $C, c>0$ such that

$$
\left|\operatorname{Tr}\left(\rho_{\infty}\left(h_{t}\right)\right)-\operatorname{Tr}\left(\rho_{\infty}\left(\tilde{h}_{t}\right)\right)\right| \leq C e^{-c / \sqrt{t}}
$$

for all $0<t \leq 1$.

Proof. For $\pi \in \Pi(G(\mathbb{R}))$ let $m_{\Gamma}(\pi)$ denote the multiplicity with which $\pi$ occurs in the discrete subspace $L_{\text {cusp }}^{2}(\Gamma \backslash G(\mathbb{R}))$ and denote by $\lambda_{\pi}$ the Casimir eigenvalue $\pi(\Omega)$ of $\pi$. For every $\pi \in \Pi(G(\mathbb{R}))$ with $m_{\Gamma}(\pi) \neq 0$ we choose an orthonormal basis $e_{\pi, i}$, $i \in I(\pi)$, for the finite-dimensional vector space $\left(\mathcal{H}(\pi) \otimes V_{\sigma}\right)^{K_{\infty}}$. Let $\Delta_{G}$ be the Laplace operator on $G(\mathbb{R})$ with respect to the left invariant metric. Note that

$$
\Delta_{G}=R\left(-\Omega+2 \Omega_{K_{\infty}}\right),
$$

where $\Omega_{K_{\infty}}$ is the Casimir element of $K_{\infty}$. Let $\lambda_{\sigma}$ be the Casimir eigenvalue of $\sigma$. We note that

$$
-\lambda_{\pi}+2 \lambda_{\sigma} \geq 0
$$

for all $\pi \in \Pi(G(\mathbb{R}))$ with $\left(\mathcal{H}(\pi) \otimes V_{\sigma}\right)^{K_{\infty}} \neq\{0\}$ (cf. Lemma 2.6 of $\left.[\mathrm{DH}]\right)$. Let $\chi_{\sigma}$ be the character of $\sigma$ and let $d=\operatorname{dim} X$. Then for every $f \in \mathcal{C}^{1}(G(\mathbb{R}))$ with $f=f * \bar{\chi}_{\sigma}$ we get

$$
\begin{aligned}
\operatorname{Tr} \rho_{\infty}(f)= & \sum_{\pi \in \Pi(G(\mathbb{R}))} m_{\Gamma}(\pi) \sum_{i \in I(\pi)}\left(\rho_{\infty}(f) e_{\pi, i}, e_{\pi, i}\right) \\
= & \sum_{\pi \in \Pi(G(\mathbb{R}))} m_{\Gamma}(\pi)\left(1-\lambda_{\pi}+2 \lambda_{\sigma}\right)^{-4 d} \\
& \times \sum_{i \in I(\pi)} \int_{G(\mathbb{R})}\left(\left(\mathrm{Id}+\Delta_{G}\right)^{4 d} f\right)(g)\left(\rho_{\infty}(g) e_{\pi, i}, e_{\pi, i}\right) d g .
\end{aligned}
$$


By [Mu1, Theorem 0.1] we have

$$
\sum_{\pi \in \Pi(G(\mathbb{R}))} m_{\Gamma}(\pi) \operatorname{dim}\left(\mathcal{H}(\pi) \otimes V_{\sigma}\right)^{K_{\infty}}\left(1-\lambda_{\pi}+2 \lambda_{\sigma}\right)^{-4 d}<\infty .
$$

Using this result we get

$$
\left|\operatorname{Tr} \rho_{\infty}(f)\right| \leq C\left\|\left(\operatorname{Id}+\Delta_{G}\right)^{4 d} f\right\|_{L^{1}(G(\mathbb{R}))}
$$

for some constant $C>0$, independent of $f$. Let $\psi_{t}=1-\varphi_{t}, t>0$. Then it follows from (4.1) that

$$
\begin{aligned}
\left|\operatorname{Tr}\left(\rho_{\infty}\left(h_{t}\right)\right)-\operatorname{Tr}\left(\rho_{\infty}\left(\tilde{h}_{t}\right)\right)\right| & =\mid \operatorname{Tr}\left(\rho_{\infty}\left(\psi_{t} h_{t}\right) \mid\right. \\
& \leq C\left\|\left(\operatorname{Id}+\Delta_{G}\right)^{4 d}\left(\psi_{t} h_{t}\right)\right\|_{L^{1}(G(\mathbb{R}))} .
\end{aligned}
$$

It remains to estimate the right hand side. Let $X_{1}, \ldots, X_{a}$ be an orthonormal basis of $\mathfrak{g}(\mathbb{R})$. Then $\Delta_{G}=-\sum_{i} X_{i}^{2}$. Denote by $\nabla$ the canonical left invariant connection on $G(\mathbb{R})$. Then it follows that there exists $C_{1}>0$ such that for all $f \in C^{\infty}(G(\mathbb{R}))$ we have

$$
\left|\left(\mathrm{Id}+\Delta_{G}\right)^{4 d} f(g)\right| \leq C_{1} \sum_{j=0}^{8 d}\left\|\nabla^{j} f(g)\right\|, \quad g \in G(\mathbb{R}) .
$$

By Proposition 3.1 there exist constants $C, c>0$ such that

$$
\left\|\nabla^{j} h_{t}(g)\right\| \leq C t^{-(a+j) / 2} e^{-c r^{2}(g) / t}, \quad g \in G(\mathbb{R}),
$$

for $j \leq 8 d$ and $0<t \leq 1$. Let $\chi_{t}$ be the characteristic function of the complement of the interval $\left(-t^{1 / 4} / \sqrt{2}, t^{1 / 4} / \sqrt{2}\right)$ in $\mathbb{R}$. Recall that $\psi_{t}(g)=(1-\varphi)\left(r^{2}(g) / t^{1 / 2}\right)$ and $(1-\varphi)(u)$ is constant for $|u| \geq 1$. This implies that there exists a constant $C_{2}>0$ such that

$$
\left\|\nabla^{j} \psi_{t}(g)\right\| \leq C_{2} t^{-4 d} \chi_{t}(r(g)), \quad g \in G(\mathbb{R}),
$$

for $j \leq 8 d$ and $0<t \leq 1$. Combining (4.3) and (4.4) we obtain

$$
\begin{aligned}
\sum_{j=0}^{8 d}\left\|\nabla^{j}\left(\psi_{t} h_{t}\right)(g)\right\| & \leq C_{2} t^{-a / 2-8 d} \chi_{t}(r(g)) e^{-c_{2} r^{2}(g) / t} \\
& \leq C_{3} e^{-c_{3} / \sqrt{t}} e^{-c_{3} r^{2}(g)}
\end{aligned}
$$

for all $g \in G(\mathbb{R})$ and $0<t \leq 1$. Finally note that for every $c>0, e^{-c r^{2}(g)}$ is an integrable function on $G(\mathbb{R})$. Together with (4.2) and (4.3) the proof follows.

We are now ready to apply the trace formula. Given $\pi \in \Pi(G(\mathbb{R}))$, let $m_{\Gamma}(\pi, S)$ denote the multiplicity with which $\pi$ occurs in the regular representation of $G(\mathbb{R})$ on $H_{\text {cusp }}^{\Gamma, S}$. Then it follows from (3.4) that

$$
\operatorname{Tr}\left(\rho_{\infty}\left(h_{t}\right) \mid H_{\text {cusp }}^{\Gamma, S}\right)=\sum_{\pi \in \Pi(G(\mathbb{R}))} e^{t \lambda_{\pi}} m_{\Gamma}(\pi, S) \operatorname{dim} \operatorname{Hom}_{K_{\infty}}\left(\mathcal{H}(\pi), V_{\sigma}^{*}\right) .
$$


We note that the $\lambda_{\pi}$ 's are the eigenvalues of the operator which is induced by $\rho_{\infty}(\Omega) \otimes \operatorname{Id}$ in $H_{\text {cusp }}^{\Gamma}(\sigma, S)$ and $m_{\Gamma}(\pi, S) \operatorname{dim} \operatorname{Hom}_{K_{\infty}}\left(\mathcal{H}(\pi), V_{\sigma}^{*}\right)$ is the multiplicity of the corresponding eigenvalue. Let

$$
N_{\text {cusp }}^{\Gamma}(T, \sigma, S)=\sum_{\left|\lambda_{\pi}\right| \leq T} m_{\Gamma}(\pi, S) \operatorname{dim} \operatorname{Hom}_{K_{\infty}}\left(\mathcal{H}(\pi), V_{\sigma}^{*}\right) .
$$

We may now state and prove our main result.

THEOREM 4.2. Let $G$ be an almost simple, connected and simply connected algebraic group defined over $\mathbb{Q}$ such that $G(\mathbb{R})$ is non compact. Let $d_{S}$ denote the formal dimension of the Steinberg representation of $G_{S}$ and let

$$
c_{S}(\Gamma)=c\left(K_{S}\right) d_{S} \operatorname{vol}\left(K_{S}\right) .
$$

Then, if $Z_{\Gamma}=\Gamma \cap Z_{G}(\mathbb{R}) \subset K_{\infty}$ is such that $\left.\sigma\right|_{Z_{\Gamma}}=\mathrm{Id}$, we have

$$
\lim _{T \rightarrow \infty} \frac{N_{c u s p}^{\Gamma}(T, \sigma, S)}{T^{d / 2}}=c_{S}(\Gamma) \frac{\operatorname{vol}(\Gamma \backslash X)}{(4 \pi)^{d / 2} \Gamma(d / 2+1)} .
$$

Proof. We apply the trace formula to

$$
f_{t}=\tilde{h}_{t} \otimes e_{\text {fin }, S} \otimes f_{S} .
$$

The assumptions of Corollary 2.3 are satisfied. We first observe that $b\left(\tilde{h}_{t}, S\right)$ remains bounded. Since $G(\mathbb{R})$ is non compact we have

$$
\operatorname{dim}\left(G(\mathbb{R}) / K_{\infty}\right)=d>0
$$

and hence $t^{d / 2} b\left(\tilde{h}_{t}, S\right) \rightarrow 0$ as $t \rightarrow 0$. Then Corollary 2.3 yields

$$
t^{d / 2} \operatorname{tr}\left(\rho_{\infty}\left(\tilde{h}_{t}\right) \mid H_{\text {cusp }}^{\Gamma, S}\right)=c\left(K_{S}\right) t^{d / 2} \sum_{\gamma \in \mathfrak{G}_{e}} a(\gamma) \mathcal{O}_{\gamma}\left(f_{t}\right)+o(1)
$$

as $t \rightarrow 0$. Since the support of $f_{\Sigma}$ remains in a fixed compact set we already observed in Proposition 2.1 that in the sum over $\mathfrak{G}_{e}$ only a finite fixed number of terms may be non zero. Hence it suffices to estimate each term. We first remark that

$$
\mathcal{O}_{\gamma}\left(f_{t}\right)=\mathcal{O}_{\gamma}\left(\tilde{h}_{t}\right) \mathcal{O}_{\gamma}\left(e_{\text {fin }, S}\right) \mathcal{O}_{\gamma}\left(f_{S}\right) .
$$

We shall now use results that are established for the case $G=\operatorname{SL}(n)$ in [Mu2], but notation make sense and proofs extend readily for the general case. According to [Mu2, Proposition 7.3], we have

$$
t^{d / 2} J_{M}\left(\gamma, \tilde{h}_{t}\right) \rightarrow 0, \quad t \rightarrow 0,
$$

for $M=G$ and $\gamma \notin Z_{G}(\mathbb{R})$, while we know by [Mu2, Lemma 2.3] that $t^{d / 2} h_{t}(z)$ has a non zero positive limit for $z \in Z_{G}(\mathbb{R})$ :

$$
t^{d / 2} h_{t}(z) \rightarrow \frac{\operatorname{tr} \sigma(z)}{(4 \pi)^{d / 2} \operatorname{vol}\left(K_{\infty}\right)} .
$$

Since

$$
J_{G}(\gamma, h)=\mathcal{O}_{\gamma}(h)
$$


it follows that non central elements give a negligible contribution and, since $d_{S}=$ $f_{S}(z)$, we get

$$
\lim _{t \rightarrow 0} t^{d / 2} \operatorname{Tr}\left(\rho_{\infty}\left(\tilde{h}_{t}\right) \mid H_{\text {cusp }}^{\Gamma, S}\right)=\frac{\operatorname{vol}(G(\mathbb{Q}) \backslash G(\mathbb{A})) c_{S}(\Gamma)}{\operatorname{vol}\left(K_{\text {fin }}\right)} \lim _{t \rightarrow 0} t^{d / 2} \sum_{z \in Z_{\Gamma}} h_{t}(z)
$$

with

$$
c_{S}(\Gamma)=c\left(K_{S}\right) d_{S} \operatorname{vol}\left(K_{S}\right)
$$

We observe that

$$
\frac{\operatorname{vol}(G(\mathbb{Q}) \backslash G(\mathbb{A}))}{\operatorname{vol}\left(K_{\infty}\right) \operatorname{vol}\left(K_{\text {fin }}\right)}=\frac{\operatorname{vol}(\Gamma \backslash X)}{\operatorname{Card}\left(Z_{\Gamma}\right)}
$$

Using (4.6) and the assumption that $\left.\sigma\right|_{Z_{\Gamma}}=\mathrm{Id}$, it follows that the above limit can be rewritten as

$$
\lim _{t \rightarrow 0} t^{d / 2} \operatorname{tr}\left(\rho_{\infty}\left(\tilde{h}_{t}\right) \mid H_{\text {cusp }}^{\Gamma, S}\right)=c_{S}(\Gamma) \frac{\operatorname{dim} \sigma \operatorname{vol}(\Gamma \backslash X)}{(4 \pi)^{d / 2}} .
$$

Now, it follows from Proposition 4.1 that

$$
\lim _{t \rightarrow 0} t^{d / 2} \operatorname{Tr}\left(\rho_{\infty}\left(\tilde{h}_{t}\right) \mid H_{\text {cusp }}^{\Gamma, S}\right)=\lim _{t \rightarrow 0} t^{d / 2} \operatorname{Tr}\left(\rho_{\infty}\left(h_{t}\right) \mid H_{\text {cusp }}^{\Gamma, S}\right),
$$

and hence we get

$$
\lim _{t \rightarrow 0} t^{d / 2} \operatorname{Tr}\left(\rho_{\infty}\left(h_{t}\right) \mid H_{\text {cusp }}^{\Gamma, S}\right)=c_{S}(\Gamma) \frac{\operatorname{dim} \sigma \operatorname{vol}(\Gamma \backslash X)}{(4 \pi)^{d / 2}} .
$$

We conclude using (4.5) and the Tauberian theorem.

As explained in the introduction, Theorem 1.1 follows immediately from Theorem 4.2 .

Remarks. 1) The constant $c_{S}(\Gamma)$ is non zero if and only if $c\left(K_{S}\right) \neq 0$ and this is true whenever $K_{S}$ is small enough so that the space of the Steinberg representation of $G_{S}$ contains non zero $K_{S}$-invariant vectors. This is the case if $K_{p} \subset I_{p}$ a minimal parahoric subgroup for $p \in S$.

2) If the assumption $\left.\sigma\right|_{Z_{\Gamma}}=\mathrm{Id}$ in Theorem 4.2 is not satisfied, i.e, if there exists $z \in Z_{\Gamma}$ such that $\sigma(z) \neq \mathrm{Id}$, then $H_{\text {cusp }}^{\Gamma}(\sigma)=\{0\}$.

\section{REFERENCES}

[Ar] J. Arthur, The invariant trace formula II, J. Am. Math. Soc., 1 (1988), pp. 501-554.

[BM] D. Barbasch And H. Moscovici, $L^{2}$-index and the Selberg trace formula, J. Funct. Analysis, 53 (1983), pp. 151-201.

[Clo] L. Clozel, On the cohomology of Kottwitz's arithmetic varieties, Duke Math. J., 72 (1993), pp. $757-795$.

[DH] A. Deitmar and W. Hoffmann, On limit multiplicities for spaces of automorphic forms, Canad. J. Math., 51 (1999), pp. 952-976.

[Do] H. Donnelly, On the cuspidal spectrum for finite volume symmetric spaces, J. Diff. Geom., 17 (1982), pp. 239-253.

[Ef] I. Efrat, The Selberg trace formula for $\mathrm{PSL}_{2}(\mathbb{R})$, Mem. Amer. Math. Soc., 65 (1987), no. 359.

[Ko] R. Kоттwitz, Tamagawa numbers Annals of Math., 127 (1988), pp. 629-646. 
[Mil] St. Miller, On the existence and temperedness of cusp forms for $\mathrm{SL}_{3}(\mathbb{Z})$, J. Reine Angew. Math., 533 (2001), pp. 127-169.

[Mu1] W. MüLlER, The trace class conjecture in the theory of automorphic forms, Annals of Math., 130 (1989), pp. 473-529.

[Mu2] W. MüLLER, Weyl's law for the cuspidal spectrum of $S L(n)$, Preprint 2003, http://xxx.lanl.gov/math.RT/0311335.

[PS] I.I. Piatetski-Shapiro, The existence of Maass cusp forms and Kloosterman sums, In: "Theta functions-Bowdoin 1987", pp. 267-276, Proc. Sympos. Pure Math., 49, Part 2, Amer. Math. Soc., Providence, RI, 1989.

[Rez] A. REznikov, Eisenstein matrix and existence of cusp forms in rank one symmetric spaces, Geom. Funct. Anal., 3:1 (1993), pp. 79-105.

[Sa] P. Sarnak, On cusp forms, in: The Selberg trace formula and related topics, ed. by D. Hejhal et al., Contemporary Math. vol. 53, Amer. Math. Soc., 1984, pp. 393-407.

[Se] A. Selberg, Harmonic analysis, in "Collected Papers", Vol. I, Springer-Verlagf, BerlinHeidelberg-New York (1989), pp. 626-674.

[Ve] A.B. Venkov, Spectral theory of automorphic functions, A translation of Trudy Mat. Inst. Steklov. 153 (1981). Proc. Steklov Inst. Math. 1982, no. 4 (153). 
